The paracrine action of type I IFN secreted by natural IFN-producing cells (IPCs) (18) in response to $\mathrm{Cl} 13$ may also be involved. Another intriguing observation reported by Sevilla and colleagues is that $\mathrm{Cl} 13$ inhibits expression of MHC in DCs cultured from bone marrow cells, although type I IFN would be expected to increase at least MHC class I expression. Whether $\mathrm{Cl} 13$ directly inhibits MHC synthesis by mechanisms similar to those employed by herpes viruses (19) remains to be determined.

In conclusion, the $\mathrm{Cl} 13$ infection model underscores the central role of DCs in mediating viral immunosuppression and describes novel methods by which a virus can impair DCs. It will be important to investigate the influence of viral burden on these mechanisms. Infection of bone marrow and suppression of DC development may require higher viral loads than infection of peripheral DCs. Another important question is whether Cl 13 immunosuppression involves other APCs that may participate in anti-LCMV immune responses, such as IPCs and macrophages. Certainly, $\mathrm{Cl} 13$ infection will provide a valuable model to test whether increasing DC numbers, their maturation, and their $T$ cell stimulatory capacity can improve the efficacy of vaccines in chronic infections associated with immunosuppression.
Address correspondence to: Marco Colonna, Department of Pathology and Immunology, Washington University School of Medicine, Box 8118, 660 South Euclid Avenue, St. Louis, Missouri 63110, USA. Phone: (314) 362-0367; Fax: (314) 362-4096; E-mail: mcolonna@pathology.wustl.edu.

1. Oldstone, M.B. 1997. How viruses escape from cytotoxic T lymphocytes: molecular parameters and players. Virology. 234:179-185.

2. Klenerman, P., and Zinkernagel, R.M. 1998. Original antigenic sin impairs cytotoxic $\mathrm{T}$ lymphocyte responses to viruses bearing variant epitopes. Nature. 394:482-485.

3. Ciurea, A., et al. 2000. Viral persistence in vivo through selection of neutralizing antibody-escape variants. Proc. Natl. Acad. Sci. U. S. A. 97:2749-2754.

4. Sevilla, N., McGavern, D.B., Teng, C., Kunz, S., and Oldstone, M.B.A. 2004. Viral targeting of hematopoietic progenitors and inhibition of DC maturation as a dual strategy for immune subversion. J. Clin. Invest. 113:737-745. doi:10.1172/JCI200420243.

5. Ahmed, R., and Oldstone, M.B. 1988. Organ-specific selection of viral variants during chronic infection. J. Exp. Med. 167:1719-1724.

6. Sevilla, N., et al. 2000. Immunosuppression and resultant viral persistence by specific viral targeting of dendritic cells. J. Exp. Med. 192:1249-1260.

7. Banchereau, J., and Steinman, R.M. 1998. Dendritic cells and the control of immunity. Nature. 392:245-252.

8. Sevilla, N., Kunz, S., McGavern, D., and Oldstone, M.B. 2003. Infection of dendritic cells by lymphocytic choriomeningitis virus. Curr. Top. Microbiol. Immunol. 276:125-144.

9. Servet-Delprat, C., Vidalain, P.O., Valentin, H., and Rabourdin-Combe, C. 2003. Measles virus and den- dritic cell functions: how specific response cohabits with immunosuppression. Curr. Top. Microbiol. Immunol. 276:103-123.

10. Karp, C.L., et al. 1996. Mechanism of suppression of cell-mediated immunity by measles virus. Science. 273:228-231.

11. Salio, M., Cella, M., Suter, M., and Lanzavecchia, A. 1999. Inhibition of dendritic cell maturation by herpes simplex virus. Eur. J. Immunol. 29:3245-3253.

12. Engelmayer, J., et al. 1999. Vaccinia virus inhibits the maturation of human dendritic cells: a novel mechanism of immune evasion. J. Immunol. 163:6762-6768.

13. Andrews, D.M., Andoniou, C.E., Granucci, F., Ricciardi-Castagnoli, P., and Degli-Esposti, M.A. 2001. Infection of dendritic cells by murine cytomegalovirus induces functional paralysis. Nat. Immunol. 2:1077-1084.

14. Steinman, R.M., et al. 2003. The interaction of immunodeficiency viruses with dendritic cells. Curr. Top. Microbiol. Immunol. 276:1-30.

15. Li, L., et al. 2002. Epstein-Barr virus inhibits the development of dendritic cells by promoting apoptosis of their monocyte precursors in the presence of granulocyte macrophage-colony-stimulating factor and interleukin-4. Blood. 99:3725-3734.

16. Odermatt, B., Eppler, M., Leist, T.P., Hengartner, H., and Zinkernagel, R.M. 1991. Virus-triggered acquired immunodeficiency by cytotoxic T-cell-dependent destruction of antigen-presenting cells and lymph follicle structure. Proc. Natl. Acad. Sci. U. S. A. 88:8252-8256.

17. Diebold, S.S., et al. 2003. Viral infection switches nonplasmacytoid dendritic cells into high interferon producers. Nature. 424:324-328.

18. Colonna, M., Krug, A., and Cella, M. 2002. Interferonproducing cells: on the front line in immune responses against pathogens. Curr. Opin. Immunol. 14:373-379.

19. Tortorella, D., Gewurz, B.E., Furman, M.H., Schust, D.J., and Ploegh, H.L. 2000. Viral subversion of the immune system. Annu. Rev. Immunol.18:861-926.

\title{
The anatomy of an arrhythmia
}

\author{
Robert F. Gilmour, Jr.
}

\author{
Department of Biomedical Sciences, Cornell University, Ithaca, New York, USA.
}

\begin{abstract}
Computer simulations are potentially effective approaches to unraveling the causes of lethal heart rhythm disorders. In this issue of the JCI, Xie et al. (see the related article beginning on page 686) have embedded a well-characterized dynamic mechanism for arrhythmia development in an anatomically realistic computer model of the heart. Their demonstration that this simple mechanism governs the behavior of the complex model may provide a new target for strategies to prevent sudden death.
\end{abstract}

\section{Ventricular fibrillation and sudden cardiac death}

The primary mechanical event in the heart-the development of contractile force-is triggered by an electrical event, the

Nonstandard abbreviations used: action potential duration (APD); atrioventricular (AV); conduction velocity (CV); sinoatrial (SA); ventricular fibrillation (VF). Conflict of interest: The author has declared that no conflict of interest exists.

Citation for this article:

J. Clin. Invest. 113:662-664 (2004).

doi:10.1172/JCI200421223. cardiac action potential, through the process of excitation-contraction coupling. For the heart to contract efficiently and continuously over the life span of an individual, which may encompass many millions of heartbeats, electrical activation of the heart must occur repetitively in the proper sequence. Orderly electrical activation is accomplished by the sequential propagation of action potentials along the anatomically defined structures shown in the right panel of Figure 1. The heartbeat begins in the sinoatrial (SA) node with a sponta- neously generated action potential. Propagation of the SA nodal impulse creates wavefronts of electrical excitation that initially spread outward to atrial myocardium and then converge before crossing the atrioventricular (AV) node and entering the specialized conducting system, which consists of the bundle branches and an arborizing network of Purkinje cells. The Purkinje system then distributes activation rapidly and widely to ventricular myocardium.

If the sequence of electrical activation becomes disorganized, the mechanical activity of the heart is compromised. In the most extreme case of disorganization, ventricular fibrillation (VF), the electrical activity of the ventricles becomes so rapid and irregular that coordinated contraction ceases, causing blood pressure to plummet and death to ensue within minutes. Despite decades of intensive investigation, sudden death from 


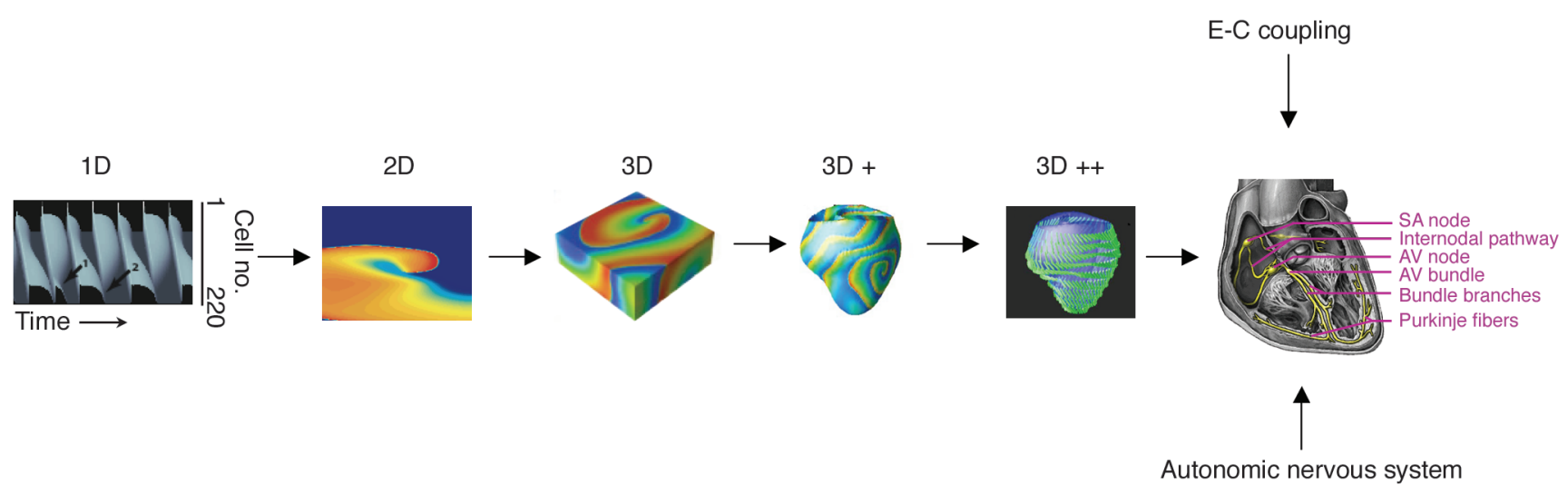

Figure 1

Evolution of cardiac computer models. The top panels illustrate, from left to right, the progression from one-dimensional (1D) models to three-dimensional (3D) models, including those that incorporate a representation of gross cardiac anatomy (3D+) and regional variations in myocardial fiber orientation (3D++). In the 1D model of a linear cardiac fiber (oriented vertically), pacing the fiber at cell 1 at a short cycle length induces discordant APD alternans in which the APD pattern at the site of stimulation is (from left to right) short APD, long APD, short APD, etc. However, the initial pattern at the other end of the fiber (cell no. 220) is the opposite: long APD followed by short APD (arrow 1). The next long APD is followed by conduction block (arrow 2). In the 2D simulation, local conduction block, indicated by the light blue region, prevents a wavefront of excitation (red area) from propagating up the right side of the sheet (where all of the cells remain in the rest state, shown in dark blue), but permits propagation up the left side, thereby inducing the formation of a spiral wave of excitation. When transferred to a 3D slab, the 2D spiral wave becomes a 3D vortex. Future challenges to creating a complete model of the heart include incorporation of excitation-contraction coupling and innervation by the sympathetic and parasympathetic limbs of the autonomic nervous system into an anatomically realistic substrate. The 1D image is reprinted with permission from Circ. Res. (8). The 3D image is reprinted with permission from Proc. Natl. Acad. Sci. U. S. A. (17). The 3D+ and 3D++ images are reprinted from Xie et al. (14). The cross-section of the heart is reproduced with permission from Benjamin/Cummings (18).

VF remains the leading cause of death in developed countries (1).

Mechanisms for ventricular fibrillation For as long as there has been an interest in defining the mechanisms underlying ventricular tachyarrhythmias, there has been the intuition that heterogeneity of cardiac electrical properties is a necessary, if not sufficient, condition for the development of such arrhythmias (2). Heterogeneity predisposes to block an electrical wavefront in one region of myocardium with continued propagation in other regions. This fractionation of the wavefront provides an opportunity for the initiation of self-sustaining reentrant excitation, manifest as conduction around an anatomical or functional obstacle (circus movement) or as a spiral wave or vortex $(2,3)$ (Figure 1). Numerous sources of heterogeneity have been identified, both in the normal (4) and in the diseased (5) heart, which presents a problem for the experimentalist and for the computer modeler when they attempt to determine which of these sources are relevant for the induction of reentry under any particular set of circumstances. The easy criticism of virtually any study conducted to date to establish a mechanistic link between one form of heterogeneity and the development of reentrant arrhythmias is "you didn't consider (fill in the blank)."
Given the plethora of potentially arrhythmogenic heterogeneities in the heart, it is remarkable that local conduction block can be accounted for, at least in certain computer models and experimental preparations, by relatively simple interactions between restitution of conduction velocity and action potential duration (6-9). In general terms, restitution refers to the recovery of some electrical property over time. For example, during pacing of a heart cell at a constant cycle length, the cardiac action potential duration (APD) and the velocity with which the action potential propagates (conduction velocity $[\mathrm{CV}]$ ) are determined by the rest, or diastolic, interval between action potentials; the greater the rest interval, the longer the subsequent $\mathrm{APD}$ and the more rapid the $\mathrm{CV}$. The restitution relations for APD and CV are typically represented by plotting either APD or CV versus the preceding diastolic interval. For reasons that have been described elsewhere (10), if the APD restitution function has a slope of 1 or more, then pacing at a short cycle length typically induces alternans, a beat-to-beat long-short alternation of APD. APD alternans and its surface ECG manifestation, QT alternans, have been linked to the development of conduction block and reentry $(6-9,11-13)$ (Figure 1).

An additional surprise from the studies of simple systems is that heterogeneity can develop from interactions between restitution of APD and CV in the complete absence of underlying intrinsic heterogeneity. In other words, the "cells" in such systems are identical in their individual behavior, yet when coupled together and perturbed by closely coupled stimuli, they develop spatially distributed dispersion of electrical properties through a purely dynamic mechanism.

The obvious question that arises with respect to such studies (at least within the cardiology community, where simplicity often is viewed with a high level of suspicion) is this: what happens when you take such simple (unidimensional, homogenous, isotropic) systems and embed them in an anatomically realistic cardiac silhouette? Will the heterogeneous anatomical features of the real heart, such as anisotropy and regional variations in fiber orientation and organization, overwhelm the contribution of dynamic heterogeneity and become the primary determinants for wavebreak and the development of reentry?

\section{Evolution of computer models of the heart}

The studies by Xie et al. in this issue of the JCI (14) were designed to answer the important questions posed above and they have done so with admirable clarity. The main result would seem to be that the precepts 
for initiation of conduction block developed using the simpler models need only be adjusted, not discarded, when considering a more anatomically complex model. This result builds on previous studies by this group and by others in which simple onedimensional models of cardiac fibers have been extended first to two-dimensional sheets of cardiac tissue and then to threedimensional slabs (Figure 1). The present study extends these models further by incorporating important aspects of gross and microscopic cardiac anatomy, including regional differences in fiber orientation. Despite the increased complexity of the anatomical substrate, the role of APD restitution in the development of VF remains intact, in that steep restitution is necessary for the initiation of wavebreak and for the disintegration of a single spiral wave into multiple wavelets (see Figures 4 and 6 from Xie et al. [ref. 14]).

Although the results of Xie et al. underscore the importance of electrical restitution for the development of VF in a more anatomically correct heart, the next set of questions inevitably arises regarding the potential impact of anatomically related electrophysiological features of the heart that were not included in their model, such as transmural and base-to-apex gradients of electrical properties, the presence of a His-Purkinje system, microscopic muscle bundle architecture, etc. The relative impact of cardiac contraction, the auto- nomic nervous system, and the anatomical, electrical, and mechanical remodeling that occur in response to the demands created by various forms of heart disease also remain to be addressed (Figure 1).

Although the prospect of accommodating all of the complexities cited above into a single model of the heart might seem daunting, such models are under development by several groups (e.g., see refs. 15, 16), and with the exponential improvement in computing power over time and the healthy interest of biologists and computer scientists, such problems promise to become tractable. It will be interesting, however, to discover how much better we understand the development of ventricular arrhythmias as layers of complexity are added. Perhaps we will be able to look back on the present state of the art and find that all of the important pieces already were in place - or perhaps not.

Address correspondence to: Robert F. Gilmour, Jr., Department of Biomedical Sciences, Cornell University, Ithaca, New York 14853-6401, USA. E-mail: rfg2@cornell.edu.

1. Zipes, D.P., and Wellens, H.J. 1998. Sudden cardiac death. Circulation. 98:2334-2351.

2. Janse, M.J. 1986. Reentry rhythms. In The heart and cardiovascular system. H.A. Fozzard, et al., editors. Raven Press. New York, New York, USA. 1203-1238.

3. Winfree, A.T. 1998. Evolving perspectives during 12 years of electrical turbulence. Chaos. 8:1-20.

4. Antzelevitch, C. 2000. Electrical heterogeneity, cardiac arrhythmias, and the sodium channel. Circ. Res. 87:964-965.
5. Janse, J., and Wit, A.L. 1989. Electrophysiological mechanisms of ventricular arrhythmias resulting from myocardial ischemia and infarction. Physiol. Rev. 69:1049-1169.

6. Watanabe, M.A., Fenton, F.H., Evans, S.J., Hastings, H.M., and Karma, A. 2001. Mechanisms for discordant alternans. J. Cardiovasc. Electrophysiol. 12:196-206.

7. Qu, Z., Garfinkel, A., Chen, P-S., and Weiss, J.N. 2000. Mechanisms of discordant alternans and induction of reentry in simulated cardiac tissue. Circulation. 102:1664-1670

8. Fox, J.J., Riccio, M.L., Hua, F., Bodenschatz, E., and Gilmour, R.F., Jr. 2002. Spatiotemporal transition to conduction block in canine ventricle. Circ. Res. 90:289-296.

9. Fox, J.J., Riccio, D.L., Drury, P., Werthman, A., and Gilmour, R.F., Jr. 2003. Dynamic mechanism for conduction block in heart tissue. New J. Phys. 5:101.1-101.14.

10. Nolasco, J.B., and Dahlen, R.W. 1968. A graphic method for the study of alternation in cardiac action potentials. J. Appl. Physiol. 25:191-196.

11. Karma, A. 1993. Spiral breakup in model equations of action potential propagation in cardiac tissue. Phys. Rev. Lett. 71:1103-1106.

12. Rosenbaum, D.S., et al. 1994. Electrical alternans and vulnerability to ventricular arrhythmias. $N$. Engl. J. Med. 330:235-241.

13. Gilmour, R.F., Jr., and Chialvo, D.R. 1999. Electrical restitution, critical mass, and the riddle of fibrillation. J. Cardiovasc. Electrophysiol. 10:1087-1089.

14. Xie, F., et al. 2004. A simulation study of the effects of cardiac anatomy in ventricular fibrillation. J. Clin. Invest. 113:686-693. doi:10.1172/JCI200417341.

15. Hunter, P.J., Pullan, A.J., and Smaill, B.H. 2003. Modeling total heart function. Annu. Rev. Biomed. Eng. 5:147-77.

16. Noble, D. 2002. Modeling the heart-from genes to cells to the whole organ. Science. 295:1678-1682.

17. Garfinkel, A., et al. 2000. Preventing ventricular fibrillation by flattening cardiac restitution. Proc. Natl. Acad. Sci. U. S. A. 97:6061-6066.

18. Mareib, E.N., and Branstrom, M.J. 1996. InterActive physiology: cardiovascular system [CD-ROM]. Benjamin/Cummings. San Francisco, California, USA.

\title{
Immune system versus tumor: shifting the balance in favor of DCs and effective immunity
}

\author{
Howard L. Kaufman'1 and Mary L. Disis ${ }^{2}$ \\ 1Department of Surgery, Columbia University, New York, New York, USA. ${ }^{2}$ Tumor Vaccine Group, University of Washington, Seattle, Washington, USA.
}

\begin{abstract}
Despite the initial excitement over cancer vaccines, the clinical effectiveness of immunotherapy has been disappointing. The suppressive milieu present within established tumors inhibits effective immune responses, although new strategies are emerging to manipulate the local tumor microenvironment and shift the balance back to a proinflammatory environment, promote DC activation, and enhance tumor immunity (see the related article beginning on page 774).
\end{abstract}

Nonstandard abbreviations used: CC chemokine receptor 7 (CCR7); human papillomavirus (HPV); Toll-like receptor (TLR).

Conflict of interest: The authors have declared that no conflict of interest exists.

Citation for this article:

J. Clin. Invest. 113:664-667 (2004).

doi:10.1172/JCI200421148.
The balance between host antitumor immunity and tumor escape

Therapeutic vaccines that have targeted established disease in cancer patients have not been successful in eliciting significant, long-lasting tumor regression. Over the last several decades most attempts to vaccinate against cancer and generate an antitumor response have been in patients with measurable tumors, and the clinical endpoint of such trials has been to evaluate a reduction in tumor burden. The inability to effectively decrease tumor growth with active immunization is most likely due, in large part, to an unfavorable tumor microenvironment incapable of propagating a robust immune response (1).

The defects in the tumor environment are many (Figure 1). The tumor itself can secrete a variety of substances that will depress or inhibit local immunity, such as IL- 6 or TGF- $\beta$, 